\title{
Mensuração da contração de polimerização de resinas compostas através da microscopia eletrônica de varredura
}

\author{
Mensuration of the polimerization shrinkage of composites through scanning electron microscope
}

Andréa de Azevedo Brito Conceição ${ }^{1}$, Ewerton Nocchi Conceição ${ }^{2}$, Darlene Dantas ${ }^{3}$, Débora Rhost ${ }^{4}$, Adriana Carboni ${ }^{5}$

1 Professora-Adjunta de Dentística da FOUFRGS. Mestre e Doutora em Dentística pela FOP/UPE

\section{Resumo}

O presente estudo avaliou três diferentes marcas comerciais de compósitos quanto à fenda resultante da contração de polimerização: Esthet-X (Dentsply); Filtek Z350 (3MESPE); Concept (Vigodent) todos na cor A3. Os compósitos foram inseridos numa matriz metálica com $7 \mathrm{~mm}$ de diâmetro interno e $2 \mathrm{~mm}$ de altura, sendo prensados entre 2 placas de vidro e fotopolimerizados por 40 segundos em uma das superfícies com aparelho de luz halógena XL 3000 (3M/ESPE) a $500+/-10 \mathrm{~mW} / \mathrm{cm}^{2}$. Após foram polidos em politriz, metalizados em ouro e levados para análise da fenda resultante da contração de polimerização em um microscópio eletrônico de varredura (Philips XL30). Foram feitas 4 medições para cada corpo de prova em áreas correspondentes a 3, 6, 9 e 12 horas. Os resultados foram submetidos à análise de variância ANOVA e teste de Tukey, ao nível de significância de $5 \%$. Os resultados mostraram os seguintes valores médios para as fendas de contração dos compósitos: Consept $(35,77 \mu \mathrm{m})$; Esthet-X $(22,01 \mu \mathrm{m})$; Filtek Z350 $(17,95 \mu \mathrm{m})$, o que demonstrou que o grupo Con diferiu estatisticamente dos demais apresentado maior valor médio em relação à fenda de contração. Baseados nos resultados obtidos, foi possível concluir que o compósito Concept apresentou maior contração de polimerização quando comparado às Resinas Compostas Esthet-X e Filtek Z350.

Palavras-chave: Resinas Compostas, Análise do estresse dentário, Propriedades físicas e químicas

\section{Abstract}

This study evaluated three different brands of composite resins on the slot resulting from the polymerization contraction: Esthet X (Dentsply); Filtek Z350 (3M / ESPE); Concept (Vigodent), all of them in color A3. Composites were placed in a metal matrix with $7 \mathrm{~mm}$ in internal diameter and $2 \mathrm{~mm}$ in height, being pressed between 2 glass plaques and photopolymerized for 40 seconds in one of the surfaces with a polymerization system by halogen light XL 3000 (3M/ ESPE) to $500+/-10 \mathrm{~mW} / \mathrm{cm}^{2}$. After that, it was polished in politriz, metallized in gold and taken for analysis of the slot resultant of the polymerization contraction in a scanning electron microscope (SEM, Philips XL30). Four measurements were made for each body of evidence in areas corresponding to 3, 6, 9 and 12 hours. The results were submitted to one-way ANOVA and Tukey's test, at the significance level of $5 \%$. The results showed the following average values for the contraction slots of composites: Concept $(35.77 \mu \mathrm{m})$; Esthet X $(22.01 \mu \mathrm{m})$; Filtek Z350 (17.95 $\mu \mathrm{m})$. It demonstrates that Con group was statistically different from the others presenting a bigger average contraction gap. Based on the results it was possible to conclude that Concept composite presented the biggest polimerization contraction when compared with Esthet-X and Filtek Z350.

Keywords: Composite resins, Dental stress analysis, Physical and chemical properties
2 Professor-Adjunto de Dentística da FOUFRGS. Mestre e Doutor em Materiais Dentários pela FOP/UNICAMP

${ }^{3}$ Professora-Adjunta de Dentística da UEPB. Mestre e Doutora em Dentística pela FOP/UPE

${ }^{4}$ Acadêmica da FO-UFRGS

${ }^{5}$ Acadêmica da FO-UFRGS

Correspondência: Andréa de Azevedo Brito Conceição

Endereço: Rua Passo da Pátria, 314/201 - Bela Vista - 90460-060

E-mail: doutora.andreabrito@ hotmail.com

\section{Introdução}

Após o surgimento das restaurações esteticamente agradáveis, com cores similares à estrutura dentária, a resina composta alcançou um lugar de destaque na odontologia restauradora. Muitos foram os avanços obtidos na evolução destes materiais. Como resultado, novos compósitos resinosos têm sido amplamente utilizados, permitindo uma grande diversificação das suas indicações com resultados estéticos e de longevidade clínica excelentes.

Apesar de ser considerado o melhor material restaurador estético direto, as resinas compostas ainda possuem desvantagens inerentes à sua formulação. Algumas destas, como desgaste e alteração de cor, já foram bastante estudadas e amenizadas. Porém, a contração de polimerização continua sendo um desafio para os fabricantes e profissionais (CARVALHO et al., 1996). A contração da resina pode comprometer a longevidade clínica das restaurações, devido ao desafio da permanência da união em áreas de estresse, como a parede cervical de cavidades proximais profundas, podendo levar a falhas precoces na restauração. Antes mesmo que os dentes restaurados sejam submetidos às cargas funcionais e às variações térmicas na cavidade bucal, já se observa tensionamento nas paredes da cavidade, devido à contração de polimerização (LABELLA, et al., 1999).

A polimerização é o processo de enrijecimento do material, em que os monômeros que fazem parte da matriz resinosa do compósito se unem por meio de ligações químicas, formando moléculas maiores, chamadas de polímeros (ANUSAVICE, 1998). Esta reação causa um estresse de contração na restauração de resina composta e possível deformidade em relação às paredes da cavidade (FEILZER, et al., 1995), podendo levar à infiltração marginal na interface dente-restauração (ASMUSSEN; PEUTZFELDT, 1998).

O grau de desenvolvimento do estresse pode ser diminuído de acordo com a forma da cavidade, o número de superfícies aderidas versus número de superfícies livres (o denominado fator $\mathrm{C}$ ), uso de materiais de base diminuindo o volume final do compósito, o tamanho, a forma e a posição de incrementos, assim como o tipo de ativador de polimerização. Os compósitos fotopolimerizados têm como principais vantagens maior tempo de trabalho, bom grau de polimerização, maior longevidade clínica e menor presença de porosidade, quando comparados aos materiais quimicamente ativados. Essas vantagens fazem com que os compósitos fotopolimerizáveis sejam superiores e indicados para a restauração de cavidades complexas (KINOMOTO et al., 1999), mesmo tendo sido constatado que apresentam maior contração de polimerização (DAVIDSON; DE GEE; FEILZER, 1984; FEILZER; DE GEE; DAVIDSON, 1993). 
Outro fator que influencia na contração de polimerização do material se refere à matriz inorgânica ou partículas de carga. A quantidade, o formato, o tamanho e a composição das partículas inorgânicas associadas aos monômeros resinosos vai determinar a quantidade de ligações duplas de carbono e o seu grau de conversão (CHEN, 2006)

Atualmente, existe uma enorme variedade de compósitos no mercado com diferentes composições, viscosidades, tipos de partículas de carga, tamanho e quantidade destas. Estudos têm mostrado que compósitos constituídos por partículas de tamanho bastante reduzido com carga submicrométrica ou nanométrica apresentam melhores resultados quanto à contração de polimerização, resistência ao desgaste e permanência do brilho superficial (CONCEIÇÃO, 2007).

O presente estudo pretende, através da microscopia eletrônica de varredura, medir a fenda de contração de polimerização proporcionada por três resinas compostas que apresentam partículas de carga e monômeros resinosos diferentes em sua composição.

\section{Materiais e Métodos}

Para o presente trabalho, foram utilizados três diferentes compósitos, divididos nos seguintes grupos $(n=8)$ : $G 1$ - Esthet $X$ (Dentsply); G2- Concept (Vigodent); G3- Filtek Z350 (3M/ ESPE).

Tabela 1. Marca comercial e Composição dos materiais avaliados.

\begin{tabular}{|c|c|c|}
\hline $\begin{array}{l}\text { Nome comercial/ } \\
\text { Fabricante }\end{array}$ & $\begin{array}{l}\text { Quantidade de Carga } \\
\text { (\%Peso), Composição, } \\
\text { Tamanho médio e } \\
\text { Classificação das } \\
\text { partículas de carga }\end{array}$ & Matriz Orgânica \\
\hline Esthet X/CaulkDentsply & $\begin{array}{c}77 \% \\
\text { Vidro de Fluorborosilica- } \\
\text { to de BarioAlumínio (0.6 } \\
\text { a } 0.8 \mu \mathrm{m}) / \text { partículas } \\
\text { nanométricas } \\
\text { de dióxido de silício (10 } \\
\text { a } 20 \text { nanômetros) } \\
\text { NanoHíbrida* }\end{array}$ & $\begin{array}{l}\text { Bis-GMA, Bis-EMA, } \\
\text { UDMA,e TEGDMA* }\end{array}$ \\
\hline Concept/Vigodent & $\begin{array}{c}77,5 \% \\
\text { silicato de bário e } \\
\text { alumínio } \\
(0,4 \mu \mathrm{m}) \\
\text { MicroHíbrida* }\end{array}$ & $\begin{array}{l}\text { Bis-GMA, UDMA, } \\
\text { Éster do ácido Metac- } \\
\text { rilato* }\end{array}$ \\
\hline Filtek Z350/3MESPE & $\begin{array}{c}78.5 \% \\
\text { Agregados de zircônia/ } \\
\text { sílica (entre } 0.6 \text { e } \\
1.4 \mu \mathrm{m} \text { ) e partículas } \\
\text { primárias de sílica não- } \\
\text { aglomerada/não-agrega- } \\
\text { da (entre 5-20nm) } \\
\text { NanoParticulada* }\end{array}$ & $\begin{array}{l}\text { BIS-GMA, UDMA, } \\
\text { BIS-EMA e TEGDMA }\end{array}$ \\
\hline
\end{tabular}

Para a confecção dos corpos de prova, foram utilizadas matrizes metálicas com $7 \mathrm{~mm}$ de diâmetro interno e $2 \mathrm{~mm}$ de altura, sendo cada uma posicionada sobre uma fita matriz de poliéster e placa de vidro. O compósito foi inserido na matriz com espátula metálica para resina em incremento único, sendo então posicionada nova fita matriz sobre a superfície da matriz preenchida por resina e pressionada uma outra placa de vidro para compressão e remoção dos excessos. Após, a placa foi removida e a ponteira de $8 \mathrm{~mm}$ de diâmetro do aparelho fotopolimerizador XL500 (3M/ESPE) foi encostada sobre a matriz de poliéster, acionando-se o mesmo por 40 segundos. A intensidade de luz permaneceu monitorada através de radiômetro (Demetron) com intensidade em torno de $500+/-10 \mathrm{~mW} / \mathrm{cm} 2$.

Foram confeccionados oito corpos de prova para cada grupo $(n=8)$, os quais foram polidos em politriz, sob irrigação com água, com lixas de carbeto de silício \# 400; \# 600; \#
1000 e \# 1200, para obter-se uma superfície lisa e polida. Sendo então levados a um aparelho de ultrassom por um tempo estimado em 10 minutos para a remoção completa de resíduos. As matrizes foram assinaladas em quatro diferentes posições com caneta Pilot, que correspondiam às posições de 3, 6, 9 e 12 horas de um relógio, na superfície da matriz mais próxima à ponta do aparelho fotopolimerizador. Os corpos de prova permaneceram por 24 horas em estufa biológica com ausência de luz e umidade para serem então metalizados em ouro. Após, foi realizada a mensuração da fenda de contração de polimerização através de um microscópio eletrônico de varredura (Philips modelo XL30), nos quatro pontos marcados previamente. A fenda foi então mensurada em micrômetros e com aumento de 2000x entre a matriz metálica e a massa do compósito. Após foi calculada uma média aritmética das quatro medidas, considerada com a medida da fenda do corpo de prova.

Os resultados foram submetidos à análise estatística através da Análise de Variância ANOVA e do teste de Tukey ao nível de $5 \%$ de significância, para verificar diferença entre a fenda da contração de polimerização de cada compósito.

\section{Resultados}

Na Tabela 1, são apresentadas as estatísticas descritivas da fenda de contração de polimerização por cada resina composta. Desta tabela é possível constatar que o valor médio mais elevado foi encontrado no grupo 2 (Concept/ Vigodent) e o menos elevado encontrado no grupo 3 (Filtek Z350/3MESPE).

Tabela 2. Estatística da fenda de contração de polimerização segundo o grupo.

\begin{tabular}{cccc}
\hline \multirow{2}{*}{ Estatística } & \multicolumn{3}{c}{ Grupo } \\
\cline { 2 - 4 } & 1 (Esthet-X) & 2 (Concept) & $3($ Z350) \\
\hline Valor mínimo $(\mathrm{mm})$ & 15,58 & 21,05 & 11,43 \\
Valor máximo $(\mathrm{mm})$ & 27,88 & 76,10 & 23,58 \\
Média $(\mathrm{mm})$ & $22,02^{\mathrm{A} *}$ & $35,78^{\mathrm{B} *}$ & $17,96^{\mathrm{A}}$ \\
Desvio padrão $(\mathrm{mm})$ & 5,02 & 17,50 & 4,01 \\
Coeficiente de variação $(\%)$ & 22,80 & 48,90 & 22,32 \\
\hline
\end{tabular}

* Letras diferentes entre os grupos significam variação estatiscamente considerável.

\section{Discussão}

As resinas compostas são constituídas por uma matriz resinosa, tendo como principal elemento o monômero Bis-GMA ou o UDMA. Sendo esses responsáveis pela porção quimicamente ativa das resinas, já que irão estabelecer ligações cruzadas durante a polimerização. Estas ligações são responsáveis pelo enrijecimento e pela resistência do material. Devido à elevada contração que sofrem estes monômeros, algumas resinas mais atuais têm apresentado o monômero Bis-EMA em sua matriz resinosa. Este, por ter maior peso molecular e menor número de ligações duplas, apresenta uma contração de polimerização menor.

Além da matriz orgânica, as partículas de carga também apresentam papel fundamental na composição das resinas e consequentemente em suas propriedades físicas e mecânicas. A maior incorporação de partículas de carga reduz a quantidade de matriz orgânica - monômeros, reduzindo a contração de polimerização (BOWEN, 1962; REES; JACOBSEN, 1989). O quartzo foi o primeiro tipo de partícula adicionada às resinas compostas, porém outros tipos de partículas já são utilizadas nas resinas mais atuais, como a sílica, por exemplo. As partículas de sílica são passíveis de microtrituração, obtendo um tamanho muito pequeno, o que permite uma maior incorporação de carga e proporciona melhores propriedades para a resina (CONDON; FERRACANE, 2000; KINOMOTO et al., 1999).

A utilização de matrizes metálicas para a medição da fenda de contração de polimerização foi observada em estudos anteriores 
(IRIE; SUZUKI; WATTS, 2002; OBICI et al., 2002; LANG, 2002). O diâmetro interno de $7 \mathrm{~mm}$ da matriz metálica permite um adequado escoamento da resina e proporciona uma contração de polimerização livre, além da similaridade entre as fendas de contração de cavidades dentarias e de teflon (IRIE; SUZUKI; WATTS, 2002). No presente estudo, o fator contração de polimerização foi estudado baseado na composição das resinas, já que não houve variação nos métodos de inserção da resina ou de fotoativação.

Foi utilizado alto vácuo para a microscopia eletrônica, já que a sua influência não é particularmente importante quando se está avaliando união adesiva entre duas estruturas distintas, como quando está se estudando sistemas adesivos unindo estrutura dental (esmalte ou dentina) e resina composta. No presente trabalho, não há nenhum desafio de união, visto que não foi utilizado sistema adesivo entre a resina composta e a superfície interna do anel metálico. Neste caso, a fenda entre os dois materiais ocorre devido à fotopolimerização da resina composta. Além disso, quatro pontos de mensuração foram utilizados para a obtenção de uma média que evitaria uma influência de algum fator desconhecido sobre a mensuração da contração de polimerização (LANG, 2002; BRESCHI et al., 2008).

Atualmente, algumas marcas comerciais de compósitos incorporaram cargas submicrométricas ou até mesmo nanométricas em seus produtos. O que, segundo alguns estudos, proporciona maior resistência ao desgaste, maior longevidade do polimento, além de menor grau de contração de polimerização (ANUSAVICE, 1998; REES; JACOBSEN, 1989) .

A resina Filtek Z350 (3M-ESPE) apresenta como principal constituinte orgânico os monômeros Bis-EMA e UDMA, com menor percentual do monômero diluente TEGDMA, além da presença de partículas de carga nanométricas em sua composição. A associação do monômero Bis-EMA partículas de carga nanométricas seria um dos prováveis fatores que levaram esta resina a apresentar a menor fenda de contração de polimerização quando comparada às resinas Esthet-X (Dentsply) e Concept (Vigodent).

A resina Esthet- $X$ também apresenta matriz orgânica próxima à resina Filtek Z350 com os monômeros Bis-EMA e UDMA em sua composição, além de uma menor incorporação de Bis-GMA. Já a sua matriz orgânica é constituída por partículas nano-híbridas e não nanométricas, como a Filtek Z350, podendo ser este o principal motivo da menor fenda de contração para a resina Filtek Z350, devido à maior incorporação de carga por volume. Já a resina Concept, apresentou o maior grau de contração de polimerização, provavelmente devido à composição de sua matriz orgânica, com forte presença dos monômeros Bis-GMA, UDMA e TEGDMA além de sua porção inorgânica composta por partículas de carga de tamanho micrométrico, o que dificulta a maior incorporação de carga por volume, como no caso das nano-híbridas/nanoparticuladas.

\section{Referências}

ANUSAVICE, K. J. Resinas para Restauração. In: ANUSAVICE, K. J. Philips Materiais Dentários. 10. ed. Rio de Janeiro: Guanabara Koogan, Cap. 12, p. 166-177, 1998.

ASMUSSEN, E.; PEUTZFELDT, A. Influence of UEDMA BisGMA and TEGMA on Selected Mechanical Properties of Experimental Resin Composites. Dent. Mater. Kidlington. v.14, n. 1, p. 51-56, Jan. 1998.

BOWEN, R. L Dental Filling Material Comprising Vinyl Silane Treated Fused Silica and a Binder Consisting of the Reaction Product of Bis Phenoland Glycidil Acrylate. US Patent 3,066,112, Nov. 271962.
BRESCHI, L. et al. Dental Adhesion Review: Aging and Stability of the Bonded Interface. Dent. Mater., Kidlington. v. 24, n. 1, p. 90-101, Jan. 2008.

CARVALHO, R. M. et al. A Review of Polymerization Contraction: The Influence of Stress Development versus Stress Relief. Oper. Dent. Seattle. v. 21. n. 1, p. 17-24, Jan./Feb. 1996.

CHEN, M. H. et al. Low Shrinkage Light Curable Nanocomposite for Dental Restorative Material. Dental Materials. Kidlington. v. 22. no. 2, p.138-145, Feb. 2006.

CONCEIÇÃO, E. N. Dentística: Saúde e Estética. 2. ed. Porto Alegre: Artmed, 2008.

CONDON, J. R.; FERRACANE, J. L. Assessing the Effect of Composite Formulation on Polymerization Stress. J. Am. Dent. Assoc., Chicago, v.131, n. 4, p. 497-503, Apr. 2000.

DAVIDSON, C. L.; DE GEE, A. J.; FEILZER, A. J. The Competition between the Composite in Bond Strength and the Polymerization Contraction Stress. J. Dent. Res., Alexandria, v. 63, n. 12, p. 1396 -1399 , Dec. 1984.

FEILZER, A. J. et al. Influence of Light Intensity on Polymerization Shrinkage and Integrity of Restorative-cavity Interface. Eur. J. Oral Sci., Copenhagen. v. 103. no. 5, p. 322-326, Oct. 1995.

FEILZER, A. J.; DE GEE, A. J.; DAVIDSON, C. L. Setting Stresses in Composites for two Diferent Curing Modes. Dent. Mater. Kidlington, v. 9. n. 1, p. 2-5, Jan. 1993.

IRIE, M.; SUZUKI, K.; WATTS, D. C. Marginal Gap Formation of Light Activated Restorative Material: Effects of Immediate Setting Shrinkage and Bond Strength. Dent. Mater. Kidlington. v. 18. n. 3. p. 203210, May 2002.

KINOMOTO, Y. et al. Comparison of Polymerization Contraction Stresses Between Self-and Light-curing Composites. J. Dent. Guildford. v. 27. n. 5. p. 383-389, July 1999.

LABELLA, R. et al. Polymerization Shrinkage Elasticity Flowable Composites and Filled Adhesives. Dent. Mater. Kidlington. v.15. n. 2, p. 128-137, Mar. 1999.

LANG, A. R. Mensuração "in vitro" da Fenda de Contração de Polimerização de Compósitos Micro-híbridos. 2002. 88f. Dissertação (Mestrado em Odontologia) Faculdade de Odontologia, Pontifícia Universidade Católica do Rio Grande do Sul, Porto Alegre, 2002.

OBICI, A. C. et al. Effect of the Photo-activation Metod on Polymerization Shrinkage of Restorative Composites. Oper. Dent. Seatle. v. 27. n. 2, p. 192-197, Mar./Apr. 2002.

REES, J. R.; JACOBSEN, P. H. The Polymerization Skrinkage of Composite Resins. Dent. Mater. Kidlington. v. 5. n. 1, p. 4144, Jan. 1989. 